Article

\title{
A Side-Absorption Concentrated Module with a Diffractive Optical Element as a Spectral-Beam-Splitter for a Hybrid-Collecting Solar System
}

\author{
An-Chi Wei ${ }^{1,2, *}$, Wei-Jie Chang ${ }^{2}$ and Jyh-Rou Sze ${ }^{3}$ \\ 1 Graduate Institute of Energy Engineering, National Central University, Taoyuan 320, Taiwan \\ 2 Department of Mechanical Engineering, National Central University, Taoyuan 320, Taiwan; \\ gmformax@gmail.com \\ 3 Instrument Technology Research Center, National Applied Research Laboratories, Hsinchu 300, Taiwan; \\ sze@narlabs.org.tw \\ * Correspondence: acwei@ncu.edu.tw; Tel.: +886-3-4267-378
}

Received: 2 November 2019; Accepted: 27 December 2019; Published: 1 January 2020

check for updates

\begin{abstract}
In this paper, we propose a side-absorption concentrated module with diffractive grating as a spectral-beam-splitter to divide sunlight into visible and infrared parts. The separate solar energy can be applied to different energy conversion devices or diverse applications, such as hybrid PV/T solar systems and other hybrid-collecting solar systems. Via the optimization of the geometric parameters of the diffractive grating, such as the grating period and height, the visible and the infrared bands can dominate the first and the zeroth diffraction orders, respectively. The designed grating integrated with the lens and the light-guide forms the proposed module, which is able to export visible and infrared light individually. This module is demonstrated in the form of an array consisting of seven units, successfully out-coupling the spectral-split beams by separate planar ports. Considering the whole solar spectrum, the simulated and measured module efficiencies of this module were $45.2 \%$ and $34.8 \%$, respectively. Analyses of the efficiency loss indicated that the improvement of the module efficiency lies in the high fill-factor lens array, the high-reflectance coating, and less scattering.
\end{abstract}

Keywords: solar concentrator; spectral beam splitting; diffractive optical element; diffractive grating

\section{Introduction}

Solar technologies have drawn significant attention due to the Earth's extreme climate and energy crises, and these technologies have recently made great progress. Mostly, those solar technologies transform sunlight into electricity or thermal power. Although one of the dominating technologies for generating electricity from sunlight is photovoltaics, this technology entails significant energy loss, including thermal loss and spectral loss. To enhance or extend the energy usage of photovoltaic systems (PV), researchers have collected waste thermal energy, such as cascading photovoltaic and thermal modules [1], integrated a PV-powered air-conditioning unit with a boiler [2], reduced the PV temperature for higher efficiency by using air-based hybrid photovoltaic/thermal systems (PV/T), water-based PV/T, and refrigerant-based PV/T [3-5], and even extended the operating bands by means of multi-junction photovoltaics, spectral-beam-splitters (SBS), and so on [6-9]. Among the systems belonging to the PV/T regime, SBS has the following advantages. Photovoltaic cells are no longer used as thermal receivers, so over-heating can be avoided. Moreover, their relatively low operation temperatures have led to high efficiency. The temperature of the heat transfer fluid (HTF) of thermal modules can be unrestricted by the operating temperature of the cells, resulting in a broad range of 
thermal applications [8]. Because of the characteristic of spectral splitting, SBS can be applied not only to the PV/T but also other hybrid-collecting solar systems, such as a dual-photovoltaic system with its cells operating in different energy-conversion bands $[10,11]$. In terms of configurations, the technique of SBS includes the following categories: dichroic filtering, liquid absorption, diffraction, and others $[8,12]$. Diffractive type of SBS can be sorted mainly by the alignment method of the sunlight receivers. The first type of alignment adopts a common optical axis for different receivers, such as photovoltaic cells and thermal tubes [13-15]. Thus, the shadow effect becomes unavoidable. The second kind of alignment arranges multiple receivers with different optical axes [16-19]. In this arrangement, the sunlight receivers can be photovoltaic cells with different absorption bands and lateral arrangements [12,13]. The third kind is proposed as a planar concentrator with a zig-zag optical axis, with the receivers located at different sides of the concentrator [20,21]. Herein, we propose a novel SBS configuration (of the third kind of alignment), consisting of a zig-zag optical axis, with integration of the diffractive optical element (DOE) and other planar optics to form a side-absorption concentrated module. In addition to the design principle of the module constructed by a single unit, we present and discuss a practical demonstration of seven units. Notably, because of its side-absorption structure, the system's thickness, complexities in component alignment, and wire connections can be reduced [22-24]. In brief, the proposed side-absorption concentrated module inherits the benefits of SBS, such as the improved operating efficiency of photovoltaics, more probable thermal-applications, etc., while its novel configuration facilitates a compact PV/T system with a simplified packaging process because of its side-absorption structure.

\section{Principles}

\subsection{Side-Absorption Concentrated Module for Spectral-Beam Splitting}

The proposed side-absorption concentrated module utilizes lenses, DOEs, and a light-guide as the condenser, the spectral beam-splitter and the out-coupling adapter, respectively. The structure of the proposed module is illustrated in Figure 1. Assume that the whole module is allocated on a solar tracker and that sunlight is regarded as normally incident to the module. When sunlight illuminates the positive lens, the light will be condensed and will pass through a DOE (considered a diffractive grating, herein). Then, the grating will diffract the light according to its wavelength. In this study, a structured light-guide with its top surface as the entrance and two side surfaces as two output ports will receive the diffracted waves, while the two ports export the sunlight in the visible and infrared bands individually. By means of this light-guide, the diffracted waves will be directed toward different output ports according to their spectra, resulting in the module splitting sunlight spectrally. Meanwhile, the proposed module inherits the advantages of the planar concentrator such that the output ports lie in the same horizontal plane, thereby allowing for planar outputs and saving the system volume. Afterwards, this module can be applied to a hybrid solar system with different energy-conversion mechanisms. As for the issues and challenges of the proposed module, they are discussed in Section 5.3 along with the comparisons to other techniques.

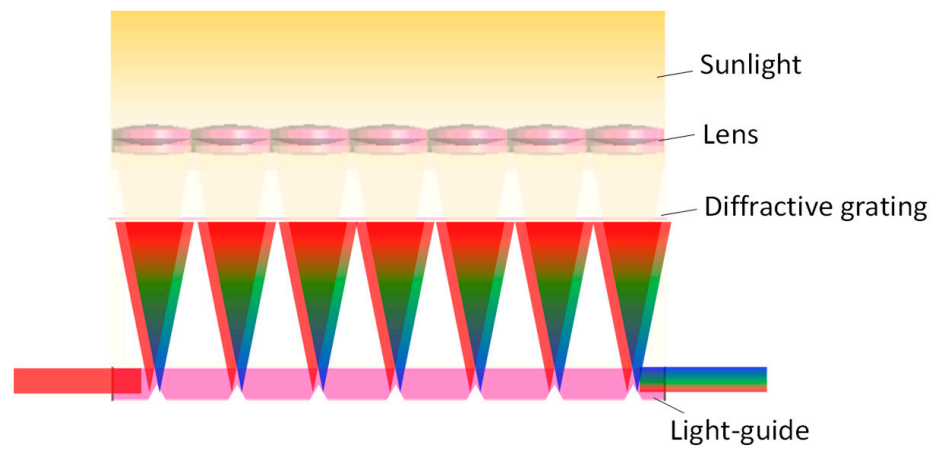

Figure 1. Schematic of the side-absorption concentrated module for spectral-beam splitting. 


\subsection{Performance Evaluation}

The evaluation of the proposed side-absorption concentrated module consists of two stages. The primary stage evaluates the diffraction performance when the light passes through the grating, and the secondary stage evaluates the out-coupling performance when the light propagates through all components and couples out from the different output ports of the light-guide. Compared to the lens, the grating is more dominant during the first stage because its dispersion affects the performance of beam-splitting, and its high-order diffraction results in a loss. Accordingly, the lens is regarded as ideal to simplify the grating analyses in the primary stage. In this study, we evaluate grating by its diffractive efficiency in the visible band, the infrared band, and the whole solar spectrum. Meanwhile, the grating is designated to diffract the visible portion of sunlight toward the first-order diffraction and the infrared portion toward the zeroth-order diffraction. The efficiency of the grating in the visible band, $\eta_{\text {grating }}^{\text {vis }}$ can be then considered as the ratio of the optical power of the first-order visible output to that of the visible sunlight:

$$
\eta_{\text {grating }}^{v i s}=\frac{\int_{\lambda_{s}^{v i s}}^{\lambda_{e}^{v i s}} \eta_{\text {diff,grating }}^{1}(\lambda) \cdot S(\lambda) d \lambda}{\int_{\lambda_{s}^{v i s}}^{\lambda_{e i s}^{v i s}} S(\lambda) d \lambda}
$$

where $\eta_{\text {diff,grating }}^{1}(\lambda)$ denotes the first-order diffraction efficiency of the grating under a source with wavelength $\lambda$, and $S$ represents the solar spectrum. $\lambda_{\mathrm{s}}{ }^{v i s}$ and $\lambda_{\mathrm{e}}{ }^{v i s}$ are the starting and ending wavelengths of the considered visible spectrum, respectively. Similarly, the grating efficiency in the infrared band, $\eta_{\text {grating }}^{I R}$ relates to the optical power of the zeroth-order infrared output:

$$
\eta_{\text {grating }}^{I R}=\frac{\int_{\lambda_{s}^{I R}}^{\lambda_{e}^{I R}} \eta_{\text {diff,grating }}^{0}(\lambda) \cdot S(\lambda) d \lambda}{\int_{\lambda_{s}^{I R}}^{\lambda_{l}^{I R}} S(\lambda) d \lambda}
$$

where $\eta_{\text {diff,grating }}^{0}(\lambda)$ denotes the zeroth-order diffraction efficiency of the grating, and $\lambda_{\mathrm{s}}{ }^{I R}$ and $\lambda_{\mathrm{e}}^{I R}$ are the start and the end wavelengths of the considered infrared spectrum, respectively. Likewise, all out-coupling waves from the designated diffraction orders are counted to derive the grating efficiency within the whole solar spectrum:

$$
\eta_{\text {grating }}^{\text {total }}=\frac{\int_{\lambda_{s}^{\text {vis }}}^{\lambda_{\text {vis }}^{\text {vis }}} \eta_{\text {diff,grating }}^{1}(\lambda) \cdot S(\lambda) d \lambda+\int_{\lambda_{s}^{I I}}^{\lambda_{l}^{I R}} \eta_{\text {diff,grating }}^{0}(\lambda) \cdot S(\lambda) d \lambda}{\int_{\lambda_{s}^{\text {total }}}^{\lambda_{\text {total }}} S(\lambda) d \lambda},
$$

where $\lambda_{\mathrm{s}}^{\text {total }}$ and $\lambda_{\mathrm{e}}^{\text {total }}$ are the start and the end wavelengths of the considered solar spectrum, respectively.

At the second stage, the synergetic effects of all components, including the lens, the grating, and the light-guide, are taken into account. The module efficiency for the visible band relates to the optical power of visible light out-coupled from the visible port of the light-guide:

$$
\eta_{\text {module }}^{\text {vis }}=\frac{\int_{\lambda_{s}^{\text {vis }}}^{\lambda_{\text {vis }}^{\text {vis }}} \eta_{\text {lens }}(\lambda) \cdot \eta_{\text {diff,grating }}^{1}(\lambda) \cdot \eta_{\text {lightguide }}(\lambda) \cdot S(\lambda) d \lambda}{\int_{\lambda_{s}^{\text {vis }}}^{\lambda_{\text {vis }}^{\text {is }}} S(\lambda) d \lambda}
$$


where $\eta_{\text {lens }}(\lambda)$ and $\eta_{\text {lightguide }}(\lambda)$ are the wavelength-dependent transmittance of the lens and the guiding efficiency of the light-guide, respectively. Similarly, the module efficiency for the infrared band and the whole solar spectrum can be respectively expressed as follows:

$$
\eta_{\text {module }}^{I R}=\frac{\int_{\lambda_{s}^{I R}}^{\lambda_{l}^{I R}} \eta_{\text {lens }}(\lambda) \cdot \eta_{\text {diff,grating }}^{0}(\lambda) \cdot \eta_{\text {lightguide }}(\lambda) \cdot S(\lambda) d \lambda}{\int_{\lambda_{s}^{I R}}^{\lambda_{l}^{I R}} S(\lambda) d \lambda}
$$

and

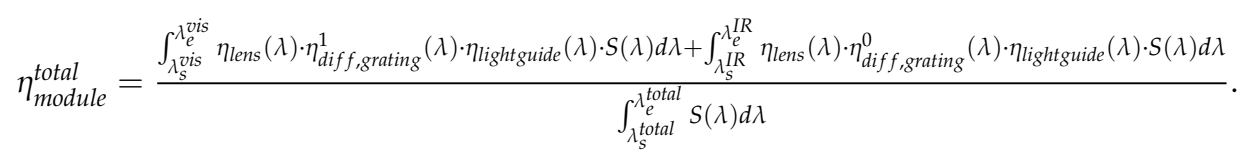

According to Equations (1)-(6), the spectral-splitting performance of the grating and that of the entire side-absorption concentrated module can be calculated and evaluated. In the following sections, these equations will be utilized for the design and optimization of the optical components in the proposed module, as well as for evaluation of the whole module.

\section{Modeling}

In order to facilitate the demonstration of a single unit and an assembled array of the proposed module, we considered the commercial specifications of lenses and then designated a lens as the condenser with a diameter of $25.4 \mathrm{~mm}$ and a distance of $30 \mathrm{~mm}$ between the lens and the grating. Since a lens with a long focal length produces a thick module, and that with a short focal length brings about large spherical aberrations, a lens with an appropriate focal length was plotted, and a commercial lens (model: AC254-080-A) with a focal length of $80 \mathrm{~mm}$ was selected. Notably, the selected lens uses achromatic coating to reduce chromatic aberration, which can simplify the optical properties of the waves incident to the grating and facilitate the grating design.

\subsection{Optimization of Diffractive Grating}

There are several considerations for designing the diffractive grating in the proposed module, including the grating period, the geometric shape, the material, and the diffraction orders in use. Because of the available fabrication processes and the requirement to achieve duplication with sufficient efficiency, the shape and material of the grating were designed as blazed and polyethylene terephthalate (PET), respectively. Meanwhile, the zeroth and the first diffraction orders were utilized. According to the grating equation, the diffractive beams can be characterized by their diffractive angles. For a normally incident light source, the diffractive angle of the $m$ th-order diffraction can be expressed as in [25]:

$$
\theta_{m}=\sin ^{-1}\left(\frac{m \lambda}{d}\right)
$$

where $d$ is the grating period. By differentiation, the angular dispersion of the grating is derived as in [26]:

$$
\frac{\partial \theta_{m}}{\partial \lambda}=\frac{m}{d \times \cos \theta_{m}} .
$$

Equation (8) indicates that diffraction order $m$ and grating period $d$ are the determinative factors of angular dispersion. In the proposed module, the diffractive angle of every spectral wave influenced the overlapping of the diffractive spots on the following light-guide. Therefore, the effects of the grating period were analyzed based on Equation (7) for every wavelength. By means of the optical tool, LightTools, the diffractive spots from a broad-band source were simulated. The results show that when the grating period was less than $16 \mu \mathrm{m}$, the overlapping of the diffractive spots was eliminated. With consideration of the fabrication accuracy, the grating period was optimized as $15 \mu \mathrm{m}$. 
Next, since the designated shape of the grating was blazed, the blaze angle was determined in terms of its diffraction efficiency, which is the merit-function to determine the blaze angle when the grating period is given [27]. Further, because of the wide spectral range of the light source, the diffraction efficiency was unable to be calculated accurately via scalar diffraction theory. Thus, the simulation was executed by means of the rigorous-coupled-wave-analysis (RCWA) software, Gsolver, to derive the diffraction efficiency of the blazed grate with different blaze angles. According to Equations (1)-(3), the grating efficiencies for the considered visible band (380-780 nm), infrared band $(780 \sim 2520 \mathrm{~nm})$, and whole solar band $(280 \sim 2520 \mathrm{~nm})$ were calculated, as shown in Figure 2 . To maximize the grating efficiency within the whole solar spectrum, the optimized blaze angle was $3.51^{\circ}$, which is equivalent to a grating height of $0.92 \mu \mathrm{m}$. Then, the maximum grating efficiency for the whole solar spectrum reached $63.3 \%$, while the corresponding efficiencies for the visible and infrared spectra were $73.8 \%$ and $54.4 \%$, respectively. Additionally, the spectral-splitting performance illustrated in Figure 3 reveals that the unused diffraction orders were greatly suppressed.

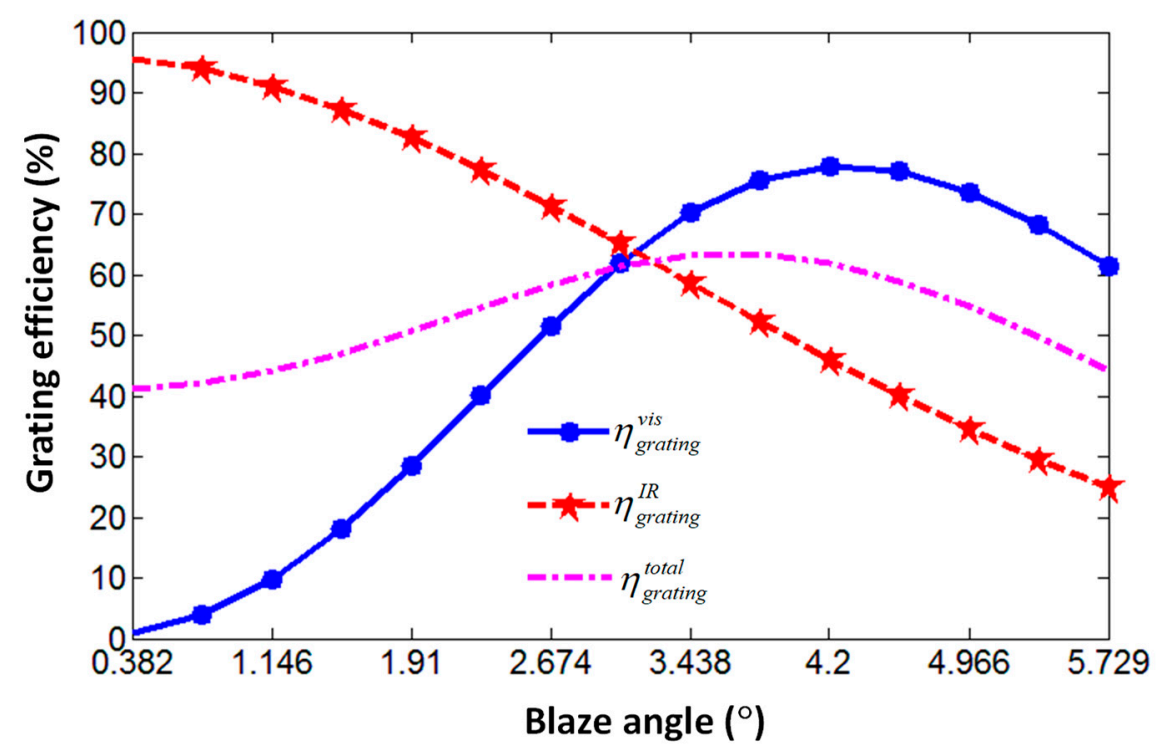

Figure 2. Relations between the grating efficiency and the blazed angle for different spectral bands.

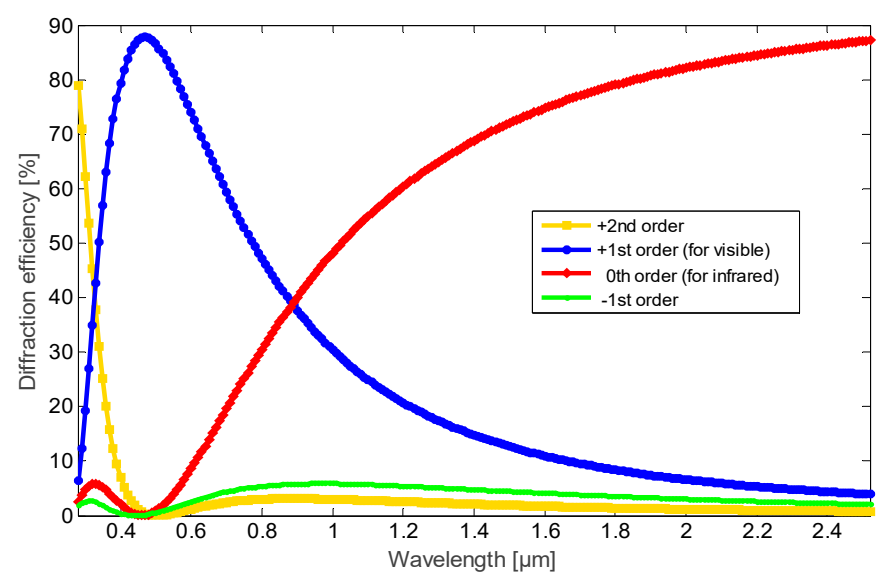

Figure 3. Diffraction efficiency for different diffraction orders in the spectral range from 280 to $2520 \mathrm{~nm}$.

\subsection{Design of the Module}

After the lens and the grating had been designed, the following step was to determine the parameters of the light-guide. Herein, a single-unit module was considered for the design. An isosceles v-groove was assumed to exist on the bottom side of the light-guide with reflective coating to direct 
the spectral-split beams toward different output ports. The ray-tracing diagram for a collimated light source impinging on the proposed module is illustrated in Figure 4. This diagram shows that, for the first-order diffraction, the spot on the light-guide shifts along the $+y$ axis when the wavelength increases, but this y-shift phenomenon does not occur for the zeroth-order light. Accordingly, adjusting the length, vertex angle, and center of the reflective v-groove, one can direct the first-order diffractive light, mainly in the visible spectrum, toward the visible output port and allow the on-axis zeroth-order diffractive light (mainly infrared) to be reflected toward the infrared output port. In order to maintain efficient propagation, the v-groove must make the light propagating within the light-guide to fulfill the condition of total internal reflection (TIR). Then, the v-groove was assigned as an isosceles triangle with a vertex angle of $120^{\circ}$ to facilitate fabrication. On the other hand, when the proposed module was constructed as an array to enlarge the solar insolation, the greater number of units resulted in longer optical paths for the rays reflected by the v-groove and a higher possibility for them to encounter other v-grooves. Subsequently, the propagation inclination of these rays changed, and the probability of rays exiting the light-guide through the entrance surface increased. In this way, more loss was induced, and the module efficiency was reduced. Thus, the geometries of the v-groove and light-guide were optimized by maximizing the module efficiency of the whole solar spectrum, as defined in Equation (6). The material of the light-guide was assigned as polymethylmethacrylate (PMMA). The simulation was performed using the software LightTools with consideration of the antireflective coating on the lens, the Fresnel loss at every interface, and the half-angle subtended by the sun, while other factors, such as the reflectance of the v-groove, were regarded as ideal. Moreover, the sizes of the grating and the light-guide in the simulation were designed according to the dimensions of the lenses, as well as the required borders and output area compatible with the receiver.

(a)

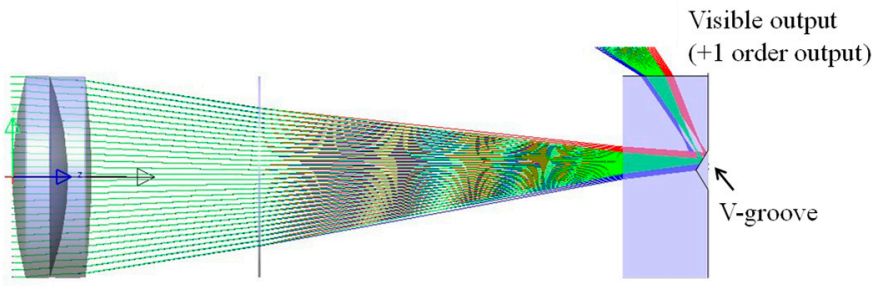

Lens Blazed grating

Light-guide

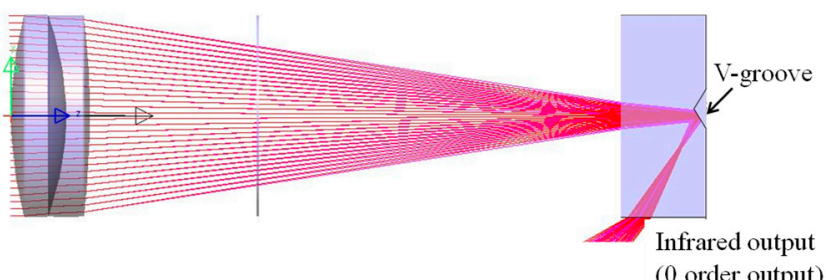

Figure 4. Single unit of the proposed module with the ray-tracing results for (a) the first-order and (b) the zeroth-order diffractive beams exiting through the visible and the infrared output ports, respectively.

Based on Equation (6), the module efficiency was counted from the simulated output power. Based on the results shown in Figure 5a, although the reflectance of the v-groove was assigned as unity, a longer v-groove length (as illustrated in Figure 5b) did not always contribute to higher module efficiency. The reason for this result is that an increased v-groove length enlarges the probability of rays encountering the second or successive grooves, resulting in more possible rays being reflected out of the light-guide through the entrance surface and leading to greater possible loss. Based on these simulated results, the geometric parameters of the v-groove were determined after the number of units for a module was given. The designed parameters of the v-groove and the light-guide will be presented in the next section along with those of the other components. 


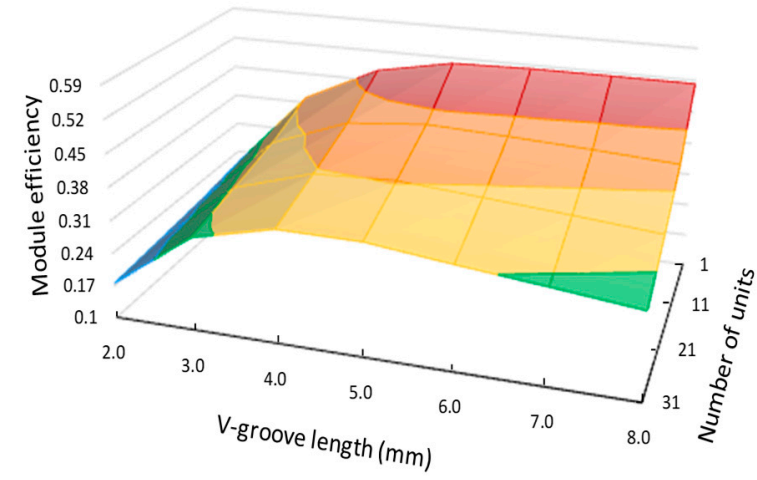

(a)

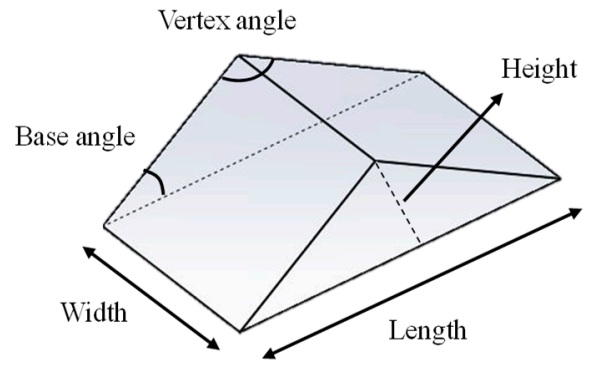

(b)

Figure 5. (a) Relation between the module efficiency for the whole solar spectrum, the length of the $\mathrm{v}$-groove, and the number of units based on the simulation results and (b) structure of the v-groove.

\section{Demonstration and Experiment}

The proposed module was demonstrated in the form of an array comprising seven units. Each unit consisted of three components, including a commercial achromatic lens (AC254-080-A), a blaze grating made of PET, and a PMMA light-guide with a carved v-groove. Based on the aforementioned design considerations, the parameters of the components were determined under the condition of a seven-unit module, as listed in Table 1. It is worth noting that the gratings of all units can be integrated into one device to simplify fabrication, and the light-guide has similar properties. Thus, the models of these two components were constructed as monolithic. Using Equations (4)-(6), the simulated module efficiency of the seven-unit module for the whole solar spectrum was $45.2 \%$, and the efficiency for the visible and the infrared output ports was $53.8 \%$ and $37.5 \%$, respectively. The grating sheet was fabricated via a roll-to-roll process. Meanwhile, the structured light-guide was fabricated by ultra-precision diamond machining, and then the carved v-grooves were coated with a reflective aluminum film. Moreover, in order to assemble all the components of the module, a fixture was designed and fabricated. The assembled module is shown in Figure 6.

After the assembly of all components with the fixture, the whole module was mounted on a dual-axis solar tracker for measurement. A customized integrating sphere, a power meter (NOVA II, Ophir Optronics), a visible spectrometer (SE1020C-025-VNIR, OtO Photonics Inc.), and an infrared spectrometer (SW2830S-050-NIRA, OtO Photonics Inc.) were utilized to measure the solar irradiance along with the out-coupling power and spectra from both the output ports. According the specifications of these instruments, the guaranteed power accuracy and the spectral accuracies of the visible and infrared bands were $\pm 3 \%$, less than $0.4 \mathrm{~nm}$, and less than $1 \mathrm{~nm}$, respectively. A photo of the experimental setup is shown in Figure 7.

Table 1. Designed parameters of the components for each unit in the 7-unit module.

\begin{tabular}{|c|c|c|c|c|c|}
\hline Lens & \multicolumn{2}{|c|}{$\begin{array}{l}\text { Diameter } \\
25.4 \mathrm{~mm}\end{array}$} & \multicolumn{2}{|c|}{$\begin{array}{l}\text { Focal Length } \\
80 \mathrm{~mm}\end{array}$} & $\begin{array}{c}\text { Material * } \\
\text { N-BK7, N-SF5 }\end{array}$ \\
\hline Grating & \multicolumn{2}{|c|}{$\begin{array}{l}\text { Period } \\
15 \mu \mathrm{m}\end{array}$} & \multicolumn{2}{|c|}{$\begin{array}{c}\text { Blaze Angle } \\
3.51^{\circ}\end{array}$} & $\begin{array}{l}\text { Material } \\
\text { PET }\end{array}$ \\
\hline Light-guide & \multicolumn{4}{|c|}{$\begin{array}{c}\text { Dimensions (Width } \times \text { Length } \times \text { Thickness) } \\
25.4 \mathrm{~mm} \times 25.4 \mathrm{~mm} \times 10.8 \mathrm{~mm}\end{array}$} & $\begin{array}{l}\text { Material } \\
\text { PMMA }\end{array}$ \\
\hline V-groove & $\begin{array}{c}\text { Vertex Angle } \\
120^{\circ}\end{array}$ & $\begin{array}{l}\text { Vertex Location } \\
\text { Off-axis } 0.82 \mathrm{~mm}\end{array}$ & $\begin{array}{l}\text { Length } \\
5 \mathrm{~mm}\end{array}$ & $\begin{array}{l}\text { Width } \\
5 \mathrm{~mm}\end{array}$ & $\begin{array}{c}\text { Coating } \\
\mathrm{Al}\end{array}$ \\
\hline
\end{tabular}

* A commercial sample treated with achromatic coating was utilized. 


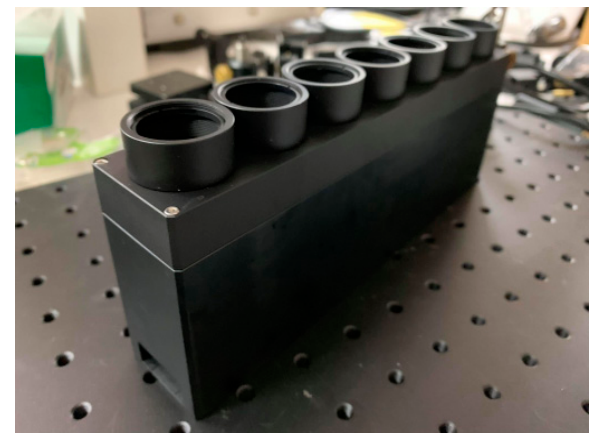

Figure 6. Photo of the demonstrated module.

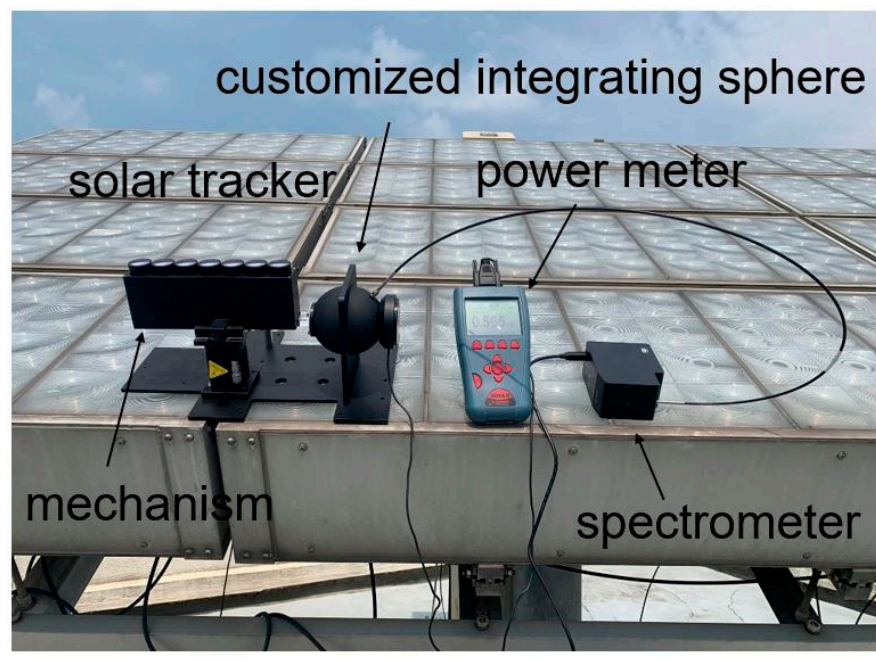

Figure 7. Assembled module with the measuring instruments allocated on the solar tracker.

The experiment was performed on a clear sunny day with the whole module fixed on the solar tracker. The irradiance of sunlight was measured as $606 \mathrm{~W} / \mathrm{m}^{2}$, and the incident solar power was counted as $2149.5 \mathrm{~mW}$. The measured power in the visible spectrum from the visible output port was $523.7 \mathrm{~mW}$, and that in the infrared spectrum from the infrared output port was $225.28 \mathrm{~mW}$. Based on Equations (4)-(5), the module efficiencies were $44.8 \%$ and $24.6 \%$ for the visible and the infrared spectra, respectively. The experimental module efficiency for the whole solar spectrum was then counted as $34.8 \%$, according to Equation (6).

When the proposed side-absorption concentrated module is connected to different receivers that are energy conversion devices, the system efficiency will vary. Ideally, the visible and the infrared output ports will be linked to the devices with complete energy conversion in the visible and infrared bands, respectively. Because of its proper absorption band and low cost, a silicon solar cell is an economic and efficient choice to link the visible output port. For the infrared port, the successive receiver for energy conversion can be either infrared solar cells or high-efficiency thermal modules, such as a solar water heater coupled with a phase-change material [28]. The system efficiencies of several feasible configurations were analyzed, and their details are reported in the following section.

\section{Analyses and Discussions}

\subsection{System Efficiency}

As mentioned above, different energy conversion devices for the proposed module will result in different system efficiencies. For example, the visible port of the proposed module can be connected to a commercial mono-crystalline silicon solar cell, with the external quantum efficiency (EQE) coarsely 
calculated as $75 \%$ [29], and the infrared port can be connected to a common water heater with a thermal efficiency of $70 \%[30,31]$. Along with the measured data of the proposed module, the system efficiency is then estimated to be $25.9 \%$.

Preferably, high-performance devices will be utilized for the proposed module. An example of such a device is an inter-digitated back contact (IBC) silicon solar cell with a high EQE [32]. This type of potential device will preferably be coupled to the visible port of the proposed module. On the other hand, a commercial germanium cell with a high EQE is one possible choice for the infrared port [33]. According to the reported EQE data for these cells and the simulated spectral responses of the proposed module, as illustrated in Figure 8, the system efficiency is calculated as $29.5 \%$. The proposed module using this combination of silicon and germanium cells is comparable in efficiency to other double-cell techniques (for example, advancing tandem cells made of silicon and perovskite $[34,35]$ ).

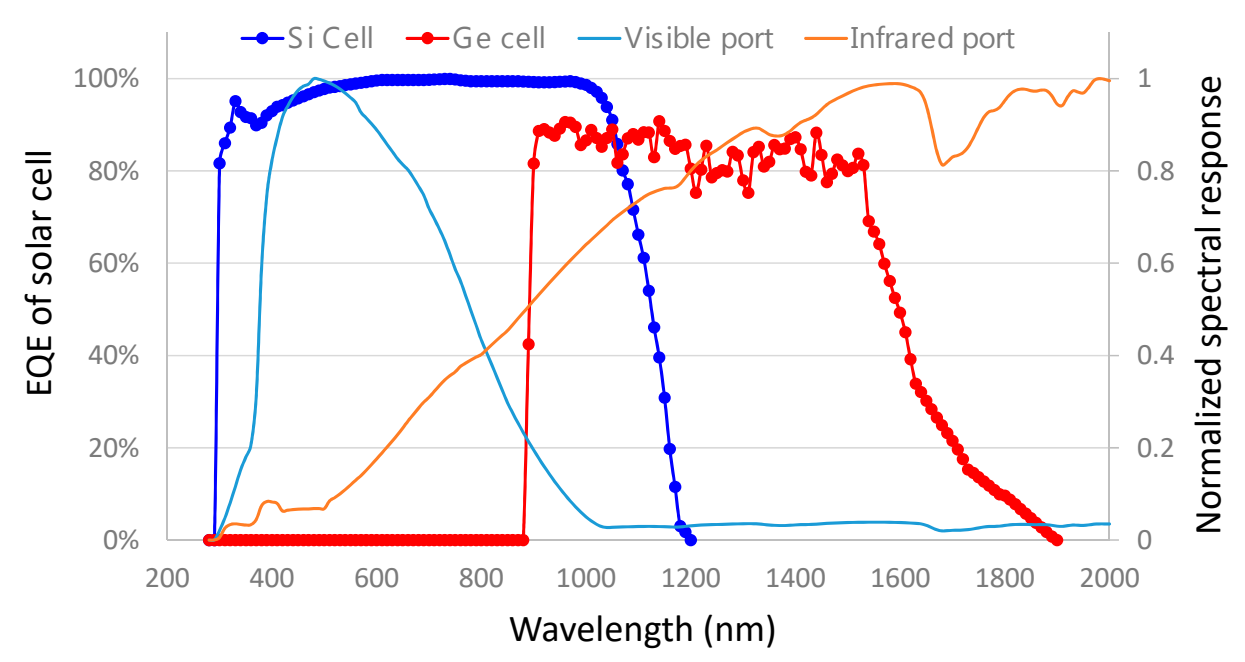

Figure 8. External quantum efficiency (EQE) data for the aforementioned silicon and germanium cells $[32,33]$ and the normalized spectral responses of the visible and infrared ports in the proposed module.

\subsection{Loss Analyses and Efficiency Enhancement}

In order to investigate the discrepancy between the simulated and measured module efficiency, we performed loss analyses via simulation. The pertinent factors are described as follows. Unlike the original design with the lenses integrated compactly, the fixture of the fabricated module produced certain spaces for mounting the lenses, as shown in Figure 9. The modeling results show that such incompactness reduced the module efficiency. Thus, a lens array designed with a high-fill-factor is preferred. Meanwhile, the reflectance of the v-groove was not as ideal as unity. Thus, the reflectance spectrum of the aluminum-coated film, as plotted in Figure 10, is not negligible. In addition, a laser beam was utilized as a testing source to examine the surface quality of the v-groove, and scattering due to the surface roughness was inspected. Based on these factors, the module efficiency was analyzed, and the results are listed in Table 2. This analysis shows that the effects of these fabrication factors on the module efficiency for the whole solar spectrum are close (each around 3\% to $4 \%$ ), and their synergy dominates a $10 \%$ loss of such efficiency.

According to the above root cause analysis of the efficiency loss, improvement of module efficiency lies in the high fill-factor for the lens array, the high-reflectance coating (such as a silver coating), and the decreased scattering through the advanced polishing process. Furthermore, through modeling, we found that the Fresnel loss occurring at each refractive interface is also a dominant factor. When all Fresnel losses are eliminated, the module efficiency over the whole solar spectrum can be increased theoretically from $45.2 \%$ to $51.2 \%$. Although producing a module without any Fresnel loss is difficult, a feasible approach for such a module would be to process two significant surfaces-the entrance 
surfaces of the grating and the light-guide-with an antireflective coating. Considering this feasible antireflective-coating, the module efficiency over the whole solar spectrum is $49.3 \%$, based on the simulation results.

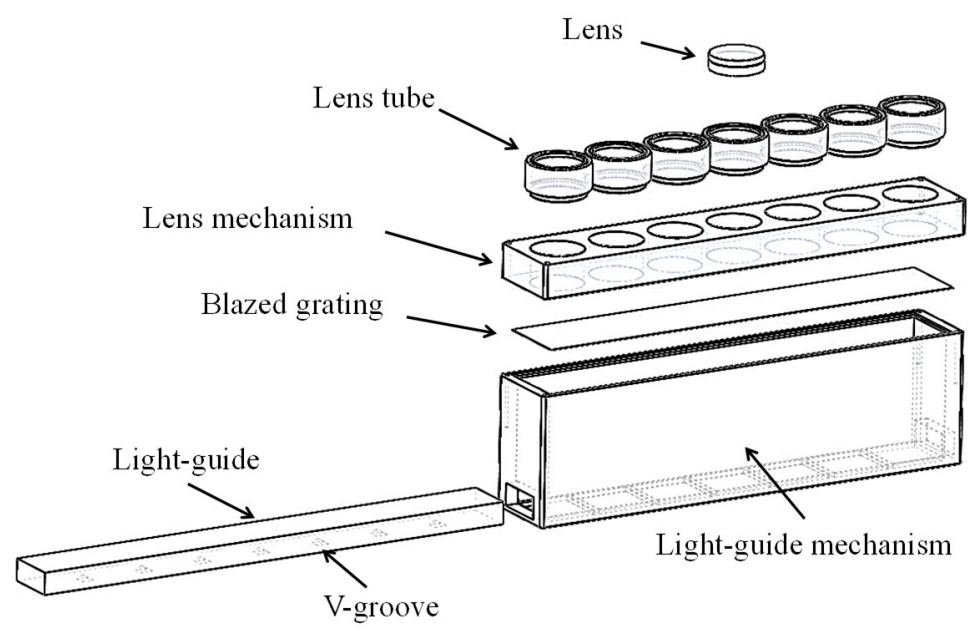

Figure 9. Exploded drawing of the practical module.

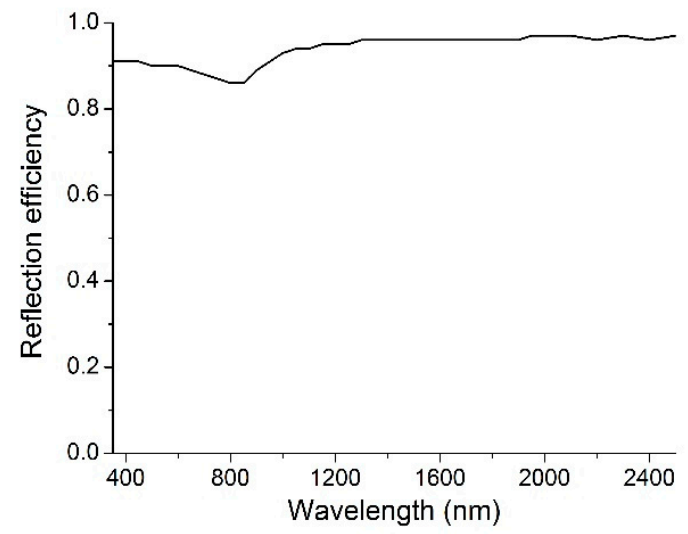

Figure 10. Spectral reflectance of the aluminum-coated film.

Table 2. Analyses of the module efficiency for a module with specific fabrication factors.

\begin{tabular}{|c|c|c|c|c|}
\hline No. & Factors & $\eta_{\text {module }}^{\text {vis }}$ & $\eta_{\text {module }}^{I R}$ & $\eta_{\text {module }}^{\text {total }}$ \\
\hline$(1)$ & Primary model ${ }^{\mathrm{a}}$ & $53.8 \%$ & $37.5 \%$ & $45.2 \%$ \\
\hline$(2)$ & $(1)+$ Bolder between lenses & $52.9 \%$ & $31.0 \%$ & $42.0 \%$ \\
\hline (3) & (2) + Reflectance of Al-coated film & $47.4 \%$ & $28.8 \%$ & $38.1 \%$ \\
\hline \multirow[t]{3}{*}{ (4) } & (3) + Scattering effect & $43.6 \%$ & $26.3 \%$ & $34.9 \%$ \\
\hline & Measurement & $44.8 \%$ & $24.6 \%$ & $34.8 \%$ \\
\hline & cted system efficiency ${ }^{b}$ & $42.8 \%$ & $14.6 \%$ & $29.5 \%$ \\
\hline
\end{tabular}

\footnotetext{
a The primary model considered the geometry and material of each component, the antireflective coating of lens, the Fresnel loss at every refractive interface, and the half angle subtended by the sun. ${ }^{\mathrm{b}}$ The expected system efficiency was calculated on the basis of model No. (4) for an embodiment with the proposed module linking assumptively to an inter-digitated back contact (IBC) silicon solar cell and a commercial germanium cell [32,33].
}

\subsection{Techniques Comparisons}

The proposed side-absorption concentrated module is a zig-zag type of diffractive SBS. Although SBS possesses the aforementioned electrical and thermal advantages, there are different issues and challenges for different categories of SBS. The dichroic filtering category requires a dichroic filter to perform spectral beam splitting [12]; however, filters made of metal suffer from a considerable 
absorption loss, while dielectric filters made from multiple thin layers generally decrease performance after receiving solar insolation for a period of time. The liquid-absorption category uses liquid to absorb the thermal portion of sunlight and cool the photovoltaics [36]. However, the flow of liquid may affect the stability of the output power of the photovoltaics. The diffractive category can realize a relatively compact structure. However, the fabrication accuracy of the diffractive element is a critical factor and relates highly to the cost.

Among the available diffractive SBS techniques, the type using a common-axis possesses a simple structure but suffers from shadowing effect and its resultant loss. The type with multiple optical axes results in a compressed system by reducing the effective length of the optical axis. Nevertheless, the energy loss occurring at the spacing between its spectrally adjacent receivers is unavoidable. The type with a zig-zag axis contributes to another compact system by laterally (rather than longitudinally) allocating the electric or thermal modules. However, the longer zig-zag path may induce more loss due to the more probability of rays encountering the successive grooves. The performance of each representative diffractive-SBS technique is listed in Table 3 for comparison. This comparison shows that the performance of the proposed module is acceptable.

Table 3. Comparisons between the different diffractive-spectral-beam-splitters (SBS) techniques.

\begin{tabular}{|c|c|c|c|}
\hline Category & Characteristics of Technique & $\begin{array}{l}\text { Module Efficiency a or } \\
\text { System Efficiency }\end{array}$ & Reference \\
\hline \multirow[t]{2}{*}{ Common axis } & $\begin{array}{l}\text { - A reflective hologram pasted } \\
\text { on the quadric surface to form a } \\
\text { spectral-beam-splitter }\end{array}$ & NA & [13] \\
\hline & $\begin{array}{l}\text { - A hologram mounted above } \\
\text { a broadband receiver, whose } \\
\text { center has an opening for } \\
\text { another spectrally selective } \\
\text { receiver }\end{array}$ & $\begin{array}{l}\text { System efficiency }=21.4 \% \text { for } \\
\text { a PV/T system }{ }^{\text {a }}\end{array}$ & [14] \\
\hline \multirow[t]{2}{*}{ Multiple optical axis } & $\begin{array}{l}\text { - A nonuniform } \\
\text { diffractive-grating as a } \\
\text { spectral-beam-splitter }\end{array}$ & $\begin{array}{l}\text { System efficiency }=34.7 \% \text { for } \\
\text { a dual-cell (InGaP/GaAs) } \\
\text { system }^{b}\end{array}$ & [17] \\
\hline & $\begin{array}{l}\text { - Micro-prism arrays as a } \\
\text { spectral-beam-splitter }\end{array}$ & $\begin{array}{l}\text { System efficiency }=46.05 \% \\
\text { for a triple-cell } \\
(\mathrm{InGaP} / \mathrm{GaAs} / \mathrm{InGaAs}) \\
\text { system }^{\mathrm{c}}\end{array}$ & [18] \\
\hline \multirow[t]{2}{*}{ Zig-zag axis } & $\begin{array}{l}\text { - An integrated } \\
\text { diffractive/refractive optical } \\
\text { element as a } \\
\text { spectral-beam-splitter, and a } \\
\text { waveguide with engraved } \\
\text { microstructures beneath the } \\
\text { spectral beam splitter }\end{array}$ & Module efficiency $\leq 55 \%{ }^{b}$ & [20] \\
\hline & $\begin{array}{l}\text { - Lenses, diffractive grating, } \\
\text { and a light-guide as the } \\
\text { condenser, the } \\
\text { spectral-beam-splitter, and the } \\
\text { out-coupling adapter, } \\
\text { respectively }\end{array}$ & $\begin{array}{l}\text { Module efficiency }=34.8 \% \mathrm{~d} \text {, } \\
\text { system efficiency }=29.5 \% \text { for } \\
\text { a dual-cell }(\mathrm{Si} / \mathrm{Ge}) \text { system }^{\mathrm{b}}\end{array}$ & Proposed \\
\hline
\end{tabular}

\footnotetext{
${ }^{a}$ Equivalent to "Optical efficiency" in some other literatures", ${ }^{b}$ simulation data, ${ }^{c}$ estimated results from the measured optical efficiency, ${ }^{\mathrm{d}}$ measured data.
}

\section{Conclusions}

In this study, we proposed a side-absorption concentrated module as a spectral-beam-splitter to separate the visible and infrared bands of sunlight for different energy-conversion applications. The proposed module integrates diffractive grating, lenses, and a light-guide to split sunlight according to spectral bands and to export the spectrum-split rays to individual planar ports. A design example with seven units has been provided for demonstration. In the simulation, the grating efficiency and 
module efficiency of this module were counted as $63 \%$ and $45.2 \%$, respectively, for the whole solar spectrum. Experimentally, the components of the proposed module were fabricated and assembled; the module efficiency for the whole solar spectrum was then measured as $34.8 \%$. Using this type of module with its visible and infrared ports linked to high-EQE silicon and germanium solar cells, respectively, a system efficiency of $29.5 \%$ is expected (based on the simulation results and the released data of solar cells). Accordingly, this system is comparable in efficiency to other double-cell systems. Moreover, the details of efficiency loss were analyzed for the proposed module, while the approaches to enhance module efficiency were discussed and presented. This analysis shows that when all Fresnel losses and the pertinent factors, including fixture limitation, reflectance of Al-coated film, and scatting effect are well-controlled, a module efficiency of $51.2 \%$ is expected for the whole solar spectrum. As for the energy gain in a PV/T system, the worthiness of the hardware development, and the payback time, they relate highly to PV materials, thermal system designs, the number of units of the proposed module, the climate and weather conditions of the demonstration site, and so on. Since some of them, such as the climate conditions, generally need sufficient time to acquire, collecting the necessary data, optimizing the performance, and lowering the cost of the proposed module will be our future researches for system realization.

Author Contributions: Conceptualization, A.-C.W.; formal analysis, A.-C.W.; funding acquisition, J.-R.S.; investigation, W.-J.C.; methodology, J.-R.S.; project administration, A.-C.W.; resources, A.-C.W.; software, W.-J.C.; supervision, A.-C.W.; validation, W.-J.C.; writing—original draft, W.-J.C.; writing—review and editing, A.-C.W. All authors have read and agreed to the published version of the manuscript.

Funding: This research was funded by the Ministry of Science and Technology, Taiwan, R.O.C., grant number MOST 107-2221-E-008-093 and 107-2221-E-492-026-MY3 by Academia Sinica, Taiwan, R.O.C., grant number AS-SS-108-04-02.

Acknowledgments: The authors thank Pi-Cheng Tung, Department of Mechanical Engineering, National Central University, Taiwan, R.O.C., and his group for the technical support on solar tracker.

Conflicts of Interest: The authors declare no conflict of interest.

\section{References}

1. Tian, Y.; Zhao, C.-Y. A review of solar collectors and thermal energy storage in solar thermal applications. Appl. Energy 2013, 104, 538-553. [CrossRef]

2. Nizetic, S.; Coko, D.; Marasovic, I. Experimental study on a hybrid energy system with small-and medium-scale applications for mild climates. Energy 2014, 75, 379-389. [CrossRef]

3. Zhang, X.; Zhao, X.; Smith, S.; Xu, J.; Yu, X. Review of R \& D progress and practical application of the solar photovoltaic/thermal (PV/T) technologies. Renew. Sustain. Energy Rev. 2012, 16, 599-617.

4. Zhao, X.; Zhang, X.; Riffat, S.; Su, X. Theoretical investigation of a novel PV/e roof module for heat pump operation. Energy Convers Manag. 2011, 52, 603-614. [CrossRef]

5. Zondag, H.; De Vries, D.; Van Helden, W.; Van Zolingen, R.; Van Steenhoven, A. The yield of different combined PV-thermal collector designs. Sol. Energy 2003, 74, 253-269. [CrossRef]

6. Yamaguchi, M.; Takamoto, T.; Araki, K.; Ekins-Daukes, N. Multi-junction III-V solar cells: Current status and future potential. Sol. Energy 2005, 79, 78-85. [CrossRef]

7. Philipps, S.P.; Dimroth, F.; Bett, A.W. High-efficiency III-V multijunction solar cells. In McEvoy's Handbook of Photovoltaics; Elsevier: Amsterdam, The Netherlands, 2018; pp. 439-472.

8. Ju, X.; Xu, C.; Han, X.; Du, X.; Wei, G.; Yang, Y. A review of the concentrated photovoltaic/thermal (CPVT) hybrid solar systems based on the spectral beam splitting technology. Appl. Energy 2017, 187, 534-563. [CrossRef]

9. Jiang, S.; Hu, P.; Mo, S.; Chen, Z. Optical modeling for a two-stage parabolic trough concentrating photovoltaic/thermal system using spectral beam splitting technology. Sol. Energy Mater. Sol. Cells 2010, 94, 1686-1696. [CrossRef] 
10. Froehlich, K.; Wagemann, E.U.; Frohn, B.; Schulat, J.; Stojanoff, C.G. Development and fabrication of a hybrid holographic solar concentrator for concurrent generation of electricity and thermal utilization. In Proceedings of the Optical Materials Technology for Energy Efficiency and Solar Energy Conversion XII, San Diego, CA, USA, 22 October 1993; pp. 311-319.

11. Fraas, L.M. Infrared Photovoltaics (IR PV) for Combined Solar Lighting and Electricity for Buildings. In Low-Cost Solar Electric Power; Springer: Berlin/Heidelberg, Germany, 2014; pp. 127-134.

12. Crisostomo, F.; Taylor, R.A.; Surjadi, D.; Mojiri, A.; Rosengarten, G.; Hawkes, E.R. Spectral splitting strategy and optical model for the development of a concentrating hybrid PV/T collector. Appl. Energy 2015, 141, 238-246. [CrossRef]

13. Meckler, M. Fixed Solar Concentrator-Collector-Satellite Receiver and Co-Generator. U.S. Patent 4,490,981, 1 January 1985.

14. Stojanoff, C.G.; Schulat, J.; Eich, M. Bandwidth-and angle-selective holographic films for solar energy applications. In Proceedings of the Solar Optical Materials XVI, Denver, CO, USA, 11 October 1999; pp. 38-49.

15. Vorndran, S.; Russo, J.M.; Wu, Y.; Gordon, M.; Kostuk, R. Holographic diffraction-through-aperture spectrum splitting for increased hybrid solar energy conversion efficiency. Int. J. Energy Res. 2015, 39, 326-335. [CrossRef]

16. Fröhlich, K.; Wagemann, E.; Schulat, H.; Schütte, H.; Stojanoff, C. Fabrication and test of a holographic concentrator for two color PVoperation. In Proceedings of the Optical Materials Technology for Energy Efficiency and Solar Energy Conversion XIII, Freiburg, Germany, 9 September 1994; pp. 812-821.

17. Stefancich, M.; Zayan, A.; Chiesa, M.; Rampino, S.; Roncati, D.; Kimerling, L.; Michel, J. Single element spectral splitting solar concentrator for multiple cells CPV system. Opt. Express 2012, 20, 9004-9018. [CrossRef] [PubMed]

18. Erim, M.N.; Erim, N.; Kurt, H. Spectral splitting for an InGaP/GaAs parallel junction solar cell. Appl. Opt. 2019, 58, 4265-4270. [CrossRef] [PubMed]

19. Li, D.; Michel, J.; Hu, J.; Gu, T. Compact spectrum splitter for laterally arrayed multi-junction concentrator photovoltaic modules. Opt. Lett. 2019, 44, 3274-3277. [CrossRef] [PubMed]

20. Kostuk, R.K.; Rosenberg, G. Analysis and design of holographic solar concentrators. In Proceedings of the High and Low Concentration for Solar Electric Applications III, San Diego, CA, USA, 9 September 2008; p. 70430I.

21. Michel, C.; Blain, P.; Clermont, L.; Languy, F.; Lenaerts, C.; Fleury-Frenette, K.; Décultot, M.; Habraken, S.; Vandormael, D.; Cloots, R. Waveguide solar concentrator design with spectrally separated light. Sol. Energy 2017, 157, 1005-1016. [CrossRef]

22. Karp, J.H.; Tremblay, E.J.; Ford, J.E. Planar micro-optic solar concentrator. Opt. Express 2010, 18, $1122-1133$. [CrossRef] [PubMed]

23. Karp, J.H.; Tremblay, E.J.; Hallas, J.M.; Ford, J.E. Orthogonal and secondary concentration in planar micro-optic solar collectors. Opt. Express 2011, 19, A673-A685. [CrossRef]

24. Wei, A.-C.; Chen, Z.-R.; Sze, J.-R. Planar solar concentrator with a v-groove array for a side-absorption concentrated photovoltaic system. Optik 2016, 127, 10858-10867. [CrossRef]

25. Hecht, E. Optics; Pearson Education: London, UK, 2017.

26. Sze, J.-R.; Wei, A.-C. Crossed Czerny-Turner Spectrometer with Extended Spectrum Using Movable Planar Mirrors. Appl. Spectrosc. 2018, 72, 776-786. [CrossRef]

27. Sinzinger, S.; Jahns, J. Microoptics; Wiley-VCH Verlag GmbH: Darmstadt, Germany, 1999.

28. Sun, L.; Xiang, N.; Yuan, Y.; Cao, X. Experimental Investigation on Performance Comparison of Solar Water Heating-Phase Change Material System and Solar Water Heating System. Energies 2019, 12, 2347. [CrossRef]

29. Solar Cell Products of United Renewable Energy. Available online: http://www.nsp.com/upload/product/ 20190606145526_battery_pdf_1.pdf (accessed on 29 October 2019).

30. Jaisankar, S.; Ananth, J.; Thulasi, S.; Jayasuthakar, S.; Sheeba, K. A comprehensive review on solar water heaters. Renew. Sustain. Energy Rev. 2011, 15, 3045-3050. [CrossRef]

31. Jamar, A.; Majid, Z.; Azmi, W.; Norhafana, M.; Razak, A. A review of water heating system for solar energy applications. Int. Commun. Heat Mass Transf. 2016, 76, 178-187. [CrossRef]

32. Haase, F.; Hollemann, C.; Schaefer, S.; Merkle, A.; Rienaecker, M.; Krügener, J.; Brendel, R.; Peibst, R. Laser contact openings for local poly-Si-metal contacts enabling 26.1\%-efficient POLO-IBC solar cells. Sol. Energy Mater. Sol. Cells 2018, 186, 184-193. [CrossRef] 
33. Solar Cell Products of Arima. Available online: http://www.arima.com.tw/group1-detail.php?index_m1_id= 1\&index_m2_id=6\&index_id=3\#parentHorizontalTab3 (accessed on 29 October 2019).

34. Bisquert, J.; Juarez-Perez, E.J. The Causes of Degradation of Perovskite Solar Cells. J. Phys. Chem. Lett. 2019, 10, 5889-5891. [CrossRef] [PubMed]

35. Zheng, J.; Mehrvarz, H.; Ma, F.-J.; Lau, C.F.J.; Green, M.A.; Huang, S.; Ho-Baillie, A.W. 21.8\% efficient monolithic perovskite/homo-junction-silicon tandem solar cell on $16 \mathrm{~cm}^{2}$. ACS Energy Lett. 2018, 3, 2299-2300. [CrossRef]

36. Matsuoka, H.; Tamura, T. Design and evaluation of thermal-photovoltaic hybrid power generation module for more efficient use of solar energy. NTT Docomo Tech. J. 2007, 12, 61-67.

(C) 2020 by the authors. Licensee MDPI, Basel, Switzerland. This article is an open access article distributed under the terms and conditions of the Creative Commons Attribution (CC BY) license (http://creativecommons.org/licenses/by/4.0/). 